\title{
ZUR ÜBERSETZUNGSÄQUIVALENZ DEUTSCHER UND POLNISCHER PHRASEOLOGISMEN MIT FARBBEZEICHNUNGEN
}

\author{
MALGORZATA PLOMIŃSKA \\ University Katowice
}

\begin{abstract}
The article is an attempt to lay down equivalents of German and Polish idiomatic phrases with colour names. The search for types of equivalence is founded upon three semantic levels of idioms as well as upon the principle of invariability in the process of translation. In this way the author tries to distinguish three types of equivalence: phraseological, lexical and zero equivalence which is prevalent in case of German and Polish phrases with colour names.
\end{abstract}

0. In linguistischen Arbeiten wird immer wieder betont, dass Phraseologismen das größte Problem des Translators sind (vgl. Hessky 1980; Zybatow 1998; Lipiński 2000). Dies resultiert aus der Komplexität des Wesens der Phraseologismen sowie aus ihren zahlreichen Funktionen, die sie auf der Textebene zu erfüllen haben. Dabei gilt für den Translator als oberstes Gebot, dass jeder Phraseologismus des Ausgangstextes (AT) im Zieltext (ZT) in einer wie auch immer gearteten phraseologischen Übersetzungsform erscheinen sollte (vgl. Zybatow 1998:153 f.). Deshalb sollte der Translator „einen schnellen Zugang zu den denkbar vielen, potentiell äquivalenten phraseologischen Ausdrücken der ZS" (Zybatow 1998:154) haben. Die deutsch-polnische phraseologische Lexokographie ist jedoch oft unvollständig - die phraseologischen Wendungen werden in den Wörterbüchern nicht immer korrekt aufgeführt, statt Äquivalenten werden Paraphrasen angegeben, viele Phraseologismen sind in die Wörterbücher gar nicht aufgenommen (vgl. Zybatow 1998:154). Daher scheint es angezeigt, neue Versuche zu unternehmen, die Übersetzungsäquivalente im Bereich der deutsch-polnischen Phraseologie systematisch und erschöpfend zu erstellen. Die Suche nach äquivalenten phraseologischen Einheiten der Ausgangssprache (AS) und der Zielsprache (ZS) ergibt sich also als zentrales Problem der konfrontativen Phraseologieforschung sowie der Übersetzungswissenschaft und -praxis. Die Aufstellung von interlingualen phraseologischen Äquivalenzbeziehungen verläuft nach E. Łabno-Falęcka (1995:216 ff.) in zwei Schritten. Der erste ist die Unterscheidung von drei Inhaltsebenen der Phraseologismen: 
- die Ebene der Bezeichnung, d.h. der direkten oder wendungsinternen Bedeutung, auf der die Phraseologismen mit ihren Strukturkomponenten ,ein Bild vermitteln",

- die Ebene der ganzheitlichen phraseologischen Bedeutung, die auch als "Idiomatizität”, , ,idiomatische Bedeutung”, „Figuriertheit" bezeichnet wird,

- die Ebene des Sinns von Phraseologismen als Textkomponenten, d.h. ihr kommunikativer Wert bzw. ihre kommunikativen Potenzen.

Der zweite Schritt bei der Suche nach äquivalenten phraseologischen Einheiten ist die Aufstellung von „Invarianzforderungen”. Dabei ist zu entscheiden, welche von den drei Inhaltsebenen der Phraseologismen invariant bleiben muss (Labno-Falęcka 1995:227). Die Äquivalenz hängt also davon ab, was beim Übersetzen invariant erhalten bleiben soll. Bei einer Übersetzung muss vom Translator erkannt werden, welche inhaltliche Schicht des Phraseologismus im AT aktualisiert wurde. Diese ist dann im Translat wiederzugeben. In den meisten Fällen muss die Ebene der ganzheitlichen Bedeutung invariant bleiben. Da jedoch auch die direkte Bedeutung in Texten relativ oft aktualisiert wird, erscheint es notwendig, phraseologische Entsprechungen der AS und der ZS nach einem kombinierten Kriterium aufzustellen, das beide inhaltlichen Ebenen berücksichtigt (Labno-Falęcka 1995:279).

Im Folgenden wird versucht, die Übersetzungsäquivalente der deutschen und polnischen Phraseologismen mit einer Farbbezeichung (FB) in Anlehung an die von E. Labno-Falęcka (1995:279 ff) vorgeschlagenen Äquivalenzbeziehungen zu erstellen. Das sprachliche Material entstammt führenden einsprachigen und zweisprachigen phraseologischen Wörterbüchern des Deutschen und des Polnischen (s. Quellen) und umfasst insgesamt 115 deutsche und 73 polnische Phraseologismen mit einer FB als Strukturkomponente.

In Anlehung an das von E. Labno-Falęcka (1995:279 ff) vorgeschlagene Kriterium zur Erstellung von phraseologischen Äquivalenten lassen sich in unserem Sprachmaterial drei Hauptypen der interlingualen phraseologischen Beziehungen aufstellen: 1. phraseologische Entsprechung, 2. lexikalische Entsprechung und 3. Nulläquivalenz, wobei der erste Typ I. weiter unterteilt werden kann.

\section{Typ 1: phraseologische Entsprechung}

1.1. Vollständige Äquivalenz, d.h. die idiomatischen Bedeutungen und die sprachlichen Bilder der Phraseologismen stimmen überein, die Komponentenbestände weisen keine Unterschiede auf. Dieser Äquivalenztyp lässt sich in unserem Sprachmaterial in 35 Fällen feststellen:

jdn. schwärzen

schwarz arbeiten

etw. in schwärzesten Farben sehen/schil- oczerniać kogoś

pracować na czarno

widzieć/przedstawiać/malować/opisywać 


\author{
dern/malen beschreiben \\ etw. schwarz sehen \\ ein schwarzer Tag \\ schwarze Gedanken \\ graue Maus \\ grauer Alltag. \\ in rosiger Laune sein \\ etw. durch eine/die rosarote/rosige Brille \\ sehen \\ jdm./etw. grünes Licht geben \\ grün und gelb vor Neid werden \\ der gelbe Neid \\ weiße Mäuse sehen \\ blau gefroren sein \\ wie ein rotes Tuch aufjdn. wirken
}

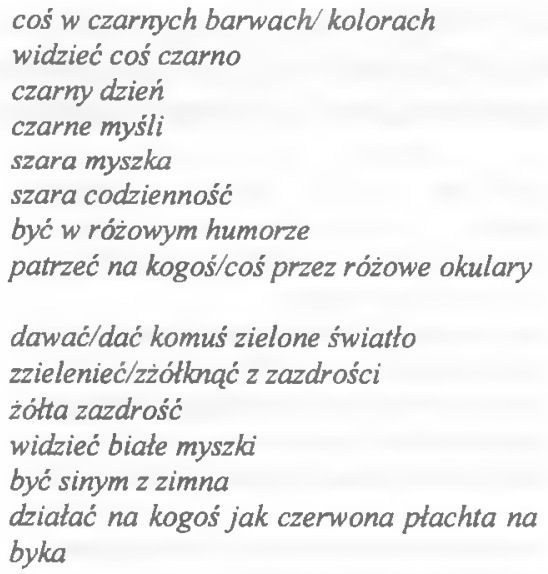

1.2. Teilweise Äquivalenz, d.h. die idiomatischen Bedeutungen stimmen überein, die sprachlichen Bilder weisen jedoch Differenzen auf. In dieser Gruppe können drei Untergruppen unterschieden werden.

1.2.1. Einem Phraseologismus mit einer FB der einen Sprache entspricht ein Phraseologismus mit einer anderen FB in der zweiten Sprache, wobei geringe strukturelle Unterschiede bestehen können. Diese Äquivalenzbeziehung konnte in unserem Material in nur 9 Fällen festgestellt werden:

\begin{abstract}
über die Grenze schwarz gehen
schwarz/grün und gelb vor Arger/Zorn werden

grün und gelb vor Neid werden

mit etw. sieht es schwarz aus

alles grau in grau malen
\end{abstract}

Grünschnabel

keine blaue/blasse Ahnung von etw. haben/keinen blauen Dunst von etw. haben

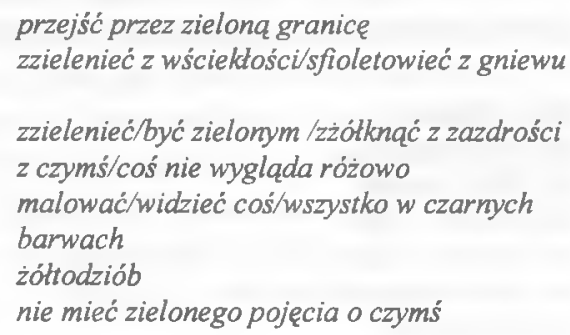

1.2.2. Einem Phraseologismus mit einer FB in der einen Sprache entspricht ein Phraseologismus mit einer anderen FB in der zweiten Sprache, wobei die sprachlichen Bilder erheblich differieren. Diese Äquivalenzbeziehung konnten wir in unserem Material in 6 Fällen feststellen:

\author{
eine schwarze Seele haben \\ ins Blaue hineinträumen \\ jdn. blau anlaufen lassen \\ grün sein \\ sich/jdn. weiß waschen wollen \\ rot sehen
}

\author{
mieć czarny charakter \\ myśleć/marzyć o niebieskich migdatach \\ zrobic kogoś na szaro \\ mieć zielono $w$ gtowie \\ wybielać się/ kogoś \\ dostać biatej goraczki
}


1.2.3. Einem Phraseologismus mit einer FB in der einen Sprache entspricht ein Phraseologismus ohne FB in der anderen Sprache. Dieser Typ phraseologischer Entsprechung trat in unserem Material in 41 Fällen auf:

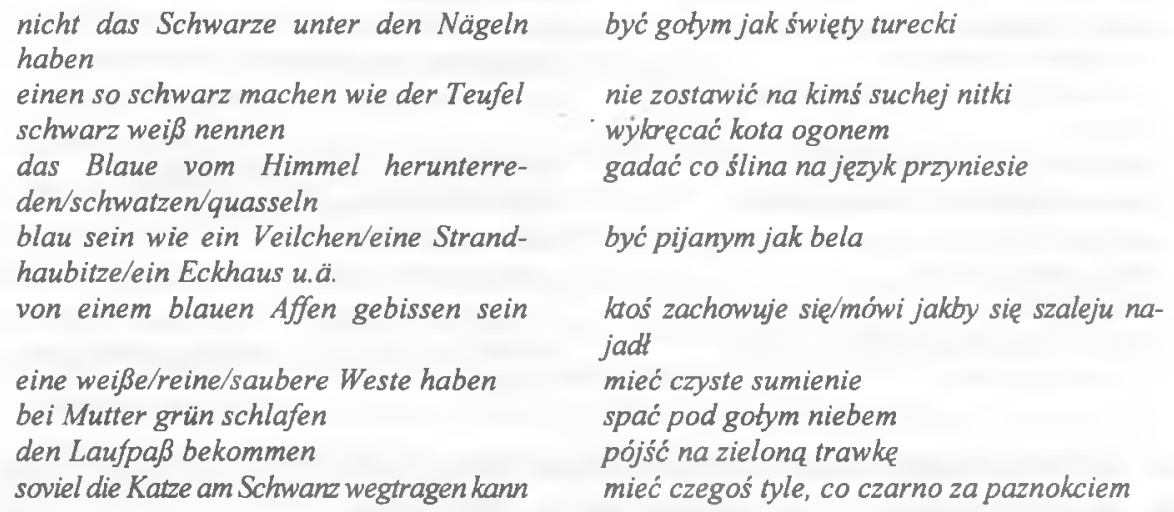

\section{Typ 2: lexikalische Entsprechung}

Im alle der lexikalischen Entsprechung stimmen die phraseologischen Bedeutungen der phraseologischen Wendungen überein, jedoch steht für einen bildhaften Phraseologismus der einen Sprache eine nicht immer bildhafte feste Wortverbindung in der anderen Sprache, und zwar meist eine Kollokation. Diese Äquivalenzbeziehung besteht in unserem Material in 6 Fällen:

$\begin{array}{ll}\text { graue Theorie } & \text { czysta teoria } \\ \text { helle Verzweiflung } & \text { czarna rozpacz } \\ \text { das Weite suchen } & \text { pójść wina dal } \\ \text { ein lockerer Vogel } & \text { niebieski ptak } \\ \text { am hellichten Tage } & \text { w biaty dzień } \\ \text { bis zum Morgengrauen/bis in den hel- } & \text { do biatego rana } \\ \text { lichten Morgen } & \end{array}$

\section{Typ 3: Nulläquivalenz}

Im Falle der Nulläquivalenz entspricht einem Phraseologismus in der einen Sprache in der anderen Sprache weder eine phraseologische noch eine feste nicht bildhafte Einheit. Dieser Äquivalenztyp lässt sich in unserem Material in 7 Fällen feststellen. Dabei stehen für einen Phraseologismus mit einer FB in der einen Sprache entweder eine Paraphrase oder ein Einzellexem in der anderen Sprache:

grün ausehen

jdm. nicht grün sein [wyglądać źle, na chorego]

[być nielubianym przez kogoś] 
grüner Junge

ins Blaue hineinreden/schießen/handeln

blau machen

jdm. den schwarzen Peter zuschieben

warten, bis man schwarz wird

keinen roten Heller für etwas geben

sich über etw. keine grauen Haare

wachsen lassen

das graue Elend kriegen

$j d m$. die gelbe Karte zeigen

[für materielle Not sparen]

[sehr schmutzig]

für jdn. kommen schlechte Zeiten, jdm.

droht Gefahr]

[über alles und alle alleine bestimmen

wollen]

[etw./jd., der unbekannt ist]

[die Jugendzeit] [niedoświadczony, niedojrzały chłopiec; łobuziak]

[mówić/robić coś bez planu, bez celu]

[bez przyczyny nie pójść do szkoły, do pracy]

[przypisywać komuś swoją winę; tak robić, by

ktoś inny zrobil coś nieprzyjemnego]

[bardzo dhugo czekać]

[nie dawać czemuś żadnych szans]

[nie martwić się czyms]

[czuć się bardzo nieszczęśliwym]

[dać komuś ostrzeżenie]

odłożyć/schować/mieć coś na czarna godzine

czarny jak święta ziemia

czarne chmury nadciagaja nad kogoś/ wiszq

nad kimś

rzadzić się jak szara gęś

biata karta

zielone lata

Die meisten deutschen und polnischen Phraseologismen mit einer FB als Strukturkomponente haben keine phraseologische Entsprechung in der anderen Sprache und stehen im Verhältnis der Nulläquivalenz ( 77 Fälle). Eine in etwa gleiche Anzahl der Phraseologismen der beiden Sprachen haben ein phraseologisches Äquivalent mit derselben FB ohne Strukturunterschiede (vollständige Äquivalenz - 35 Fälle) und ohne FB (41 Fälle im Typ 1.2.3.). Nur selten entspricht den phraseologischen Einheiten der einen Sprache in der anderen ein Phraseologismus mit derselben FB bei geringen Strukturunterschieden (9 Fälle im Typ 1.2.1.), ein Phraseologismus mit einem anderen sprachlichen Bild und einer anderen FB (6 Fälle im Typ 1.2.2.). Ebenso selten sind die lexikalischen Entsprechungen (8 Fälle). Das unterschiedliche Vorkommen der FB in den einzelnen Äquivalenztypen illustriert die nachstehende Tabelle:

\begin{tabular}{|l|c|c|c|c|}
\hline \multicolumn{1}{|c|}{ FB } & $\begin{array}{c}\text { Vollständige } \\
\text { Äquivalenz }\end{array}$ & $\begin{array}{c}\text { Teilweise } \\
\text { Äquivalenz }\end{array}$ & $\begin{array}{c}\text { Lexikalische } \\
\text { Äquivalenz }\end{array}$ & $\begin{array}{c}\text { Null- } \\
\text { Äquivalenz }\end{array}$ \\
\hline blaw/niebieski & 2 & 14 & 2 & 9 \\
(siny, btękitny) & 2 & 5 & - & 4 \\
gelb/żótty & 5 & 4 & 1 & 9 \\
graw/szary & 4 & 13 & - & 13 \\
grünzielony & 4 & 1 & - & 2 \\
rosarot/różowy & 1 & 2 & - & 21 \\
rot/czerwony & 14 & 13 & 1 & \\
schwarz/czarny & & & & \\
\hline
\end{tabular}




\begin{tabular}{|l|c|c|c|c|}
\hline FB & $\begin{array}{c}\text { Vollständige } \\
\text { Äquivalenz }\end{array}$ & $\begin{array}{c}\text { Teilweise } \\
\text { Äquivalenz }\end{array}$ & $\begin{array}{c}\text { Lexikalische } \\
\text { Äquivalenz }\end{array}$ & $\begin{array}{c}\text { Null- } \\
\text { Äquivalenz }\end{array}$ \\
\hline $\begin{array}{l}\text { weiß/biaty } \\
\text { fioletowy }\end{array}$ & 3 & 4 & 2 & 5 \\
insgesamt & - & 1 & - & - \\
\hline
\end{tabular}

Die meisten FB stehen in der Beziehung der Nulläquivalenz (ca. 41\%). Die zweitgrößte Gruppe bilden die FB im Verhältnis teilweiser Äquivalenz (ca. $30 \%$ ), die drittgrößte in der vollständigen Äquivalenz (ca. 19\%). Die kleinste Gruppe ergeben die Phraseologismen, in denen die FB als Strukturkomponente im Verhältniss der lexikalischen Entsprechung stehen (ca. 3\%). Insgesamt haben 56 (ca. 30 ) der deutschen und der polnischen Phraseologismen eine „farbige" Entsprechung in der anderen Sprache.

Farben können die menschliche Psyche beeinflussen, rufen bei Menschen Assoziationen und Emotionen hervor, deshalb werden ihnen von den Sprachgemeinschaften bestimmte Ausdruckswerte und symbolische Bedeutungen zugeschrieben (vgl. Koch 1931; Heller 1989; Kopaliński 1990; Lurker 1985). Die traditionelle, allgemeine Symbolik der Farben wird in der Phraseologie der deutschen und der polnischen Sprache genutzt, die FB beider Sprachen haben aber auch eine eigene, sprachspezifische, idiomatische Symbolik. Dabei weisen die deutschen und polnischen als phraseologische Strukturkomponente auftretenden FB sowohl Übereinstimmungen als auch Unterschiede auf.

Die größte Übereinstimmung läßt sich bei den FB schwarz und czarny, weiß und biaty, grün und zielony, gelb und żótty, rosa und różowy feststellen. Schwarz und czarny symbolisieren in den Phraseologismen beider Sprachen Unheil, Unglück, Böses, Verleumdung, Beschuldigung, Pessimismus, Schmutz, Illegalität, Heimlichkeit, z.B.:

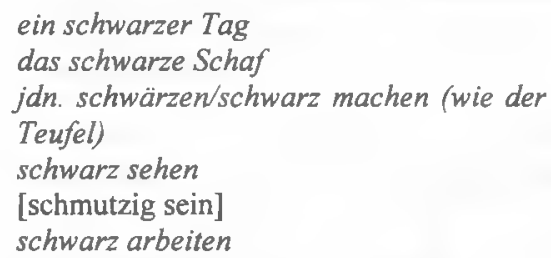

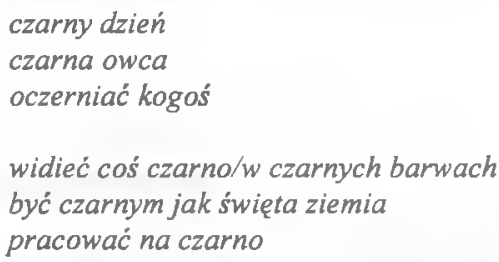

Weiß und biaty stehen für Unschuld, Anständigkeit, Aufrichtigkeit, Unbekanntes, Unbeschriebenes, z.B.:

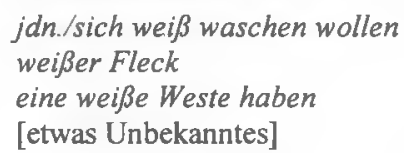

wybielać kogoś/sie biata plama

mieć czyste sumienie

biata karta 
Grün und zielony bedeuten in der deutschen und polnischen Phraseologie Vitalität, Jugendlichkeit, Unerfahrenheit, Leichtsinnigkeit, im Deutschen auch Voreiligkeit, z.B.:

grün sein

sich grün machen

[Jugendzeit]

etw. zu grün angreifen/ein Ding zu grün

abbrechen być zielonym/mieć zielono $w$ gtowie

[sprawiać wrażenie młodego, w pełni sil, brać na siebie dużo obowiązków] zielone lata

[zbyt wcześnie zacząć coś robić]

Gelb und żólty symbolisieren in der Phraseologie beider Sprachen Neid und Ärger, aber auch Jugendlichkeit und Unerfahrenheit, z.B.:

der gelbe Neid

grün und gelb vor Zorn werden

Gelbschnabel zóótta zazdrość

zżótkną́c/zzielenieć z zazdrości

żóttodziób

Die FB rosa und różowy bezeichnen Optimismus und gute Laune, z.B.:

alles in rosarotem/rosigem Licht sehen

in rosiger Laune sein widzieć/przedstwaiać coś/wszystko w różowych kolorach/w różowym świetle

być $w$ różowym humorze

Die größten Unterschiede in der idiomatischen Bedeutung der deutschen und der polnischen FB bestehen zwischen grau und szary, blau und niebieski, rot und czerwony. Grau und szary bezeichnen in beiden Sprachen Mittelmäßigkeit, Eintönigkeit und Ferne. In der deutschen Phraseologie bedeutet grau auch Sorgen, Kummer, Pessimismus, im Polnischen dagegen Betrug, z.B.:

\author{
graue Maus \\ in grauer Ferne/Zukunft \\ [ganz am Ende, als allerletzter] \\ alles grau in grau sehen/malen \\ das graue Elend kriegen \\ graue Haare kriegen \\ jdn. blau anlaufen lassen/jdm. Blaues \\ vormachen
}

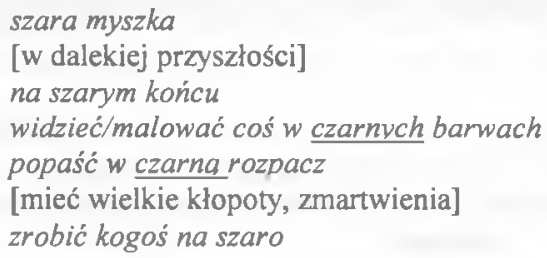

Blau und niebieski stehen in der Phraseologie beider Sprachen für sinnlose Gedanken. In deutschen Phraseologismen symbolisiert blau noch Unbekanntes, Unbestimmtes, Unangenehmes, Lüge, Betrug, niebieski in polnischen Phraseologismen dagegen Sorglosigkeit, Leichtsinnigkeit, z.B.:

ins Blaue hineinträumen eine Fahrt ins Blaue marzyć/śnić o niebieskich migdatach [podróż w nieznane] 

jdm./für jdn. das Blaue vom Himmel
(herunter) holen (wollen)/das Blaue vom
Himmel herunterlügen/jdm. blauen Dunst
vormachen
jdn. blau anlaufen lassen/jdm. Blaues
vormachen
lockerer Vogel

[okłamywać kogoś]

robić kogoś na szaro

niebieski ptak

Rot und czerwony treten in der deutschen und polnischen Phraseologie nur in ihrer direkten, nicht symbolischen Bedeutung, die auf rot gefärbte Gegenstände zurückgeht, z.B.:

\author{
wie ein rotes Tuch auf jdn. wirken \\ einer Sache rotes Licht geben \\ sich wie ein roter Faden hindurchziehen \\ sich die Augen rot weinen
}
działać na kogoś jak czerwona płachta na byka
[czemuś przeszkodzić, cofnąć pozwolenie na jakieś działania]
[być ciagle powtarzającym się, przewod- nim wątkiem w czymś]
[bardzo płakać]

Die phraseologische Bedeutung der deutschen und der polnischen FB ist zum größten Teil gleich, was sich aus dem weitgehend gemeisamen kulturellen Hintergrund der deutschen und der polnischen Sprachgemeinschaft erklären läßt. Die Unterschiede können auf historische oder kulturell bedingte Konventionen zurückgeführt werden.

Es soll hier darauf hingewiesen werden, dass die oben vorgeschlagenen Übersetzungsäquivalente der deutschen und polnischen Phraseologismen mit einer FB noch um weitere Untersuchungen unter dem Aspekt der Symbolik der einzelnen FB in der Phraseologie sowie der Einbettung dieser Phraseologismen in einem konkreten Text bzw. Kontext ergänzt werden sollten, was für die übersetzerische Praxis von großer Bedeutung wäre.

\section{QUELLEN}

Agricola, E. et al. (1970): Wörter und Wendungen. Wörterbuch zum deutschen Sprachgebrauch. München.

Bauer, W.; Dümotz, I.; Golowin, S. (1990): Lexikon der Symbole. Mythen, Symbole und Zeichen in Kultur, Religion, Kunst und Alltag. München.

Bąba, S.; Dziamska, G.; Liberek, J. (2001): Stownik frazeologiczny wspótczesnej polszczyzny.

Czochralski, J.A. (1990): Maty stownik idiomatyczny polsko-niemiecki. Warszawa.

Dittrich, H. (1970): Redensarten auf der Goldwaage. Bonn.

Donath, A. (1976): Wybór idiomów niemieckich. Warszawa.

Duden (1992): Redewendungen und sprichwörtliche Redensarten. Idiomatisches Wörterbuch der deutschen Sprache. Mannheim - Leipzig - Wien - Zürich.

Ehegötz, E. et al. (1990): Phraseologisches Wörterbuch Polnisch-Deutsch. Leipzig.

Friederich, W. (1976): Moderne deutsche Idiomatik. München. 
Głowińska, K. (2000): Popularny stownik frazeologiczny. Warszawa.

Herzog, A.; Michel, A.; Riedel, H. (1983): Deutsche idiomatische Wendungen für Ausländer. Leipzig.

Kopalinski, W. (1990): Stownik symboli. Warszawa.

Lurker, M. (1985): Wörterbuch der Symbolik. Stuttgart.

Röhrich, L. (1991): Das große Lexikon der sprichwörtlichen Redensarten. Basel - Wien.

Schemann, H. (1993): Deutsche Idiomatik. Stuttgart - Dresden.

Skorupka, S. (1985): Stownik frazeologiczny języka polskiego. Warszawa.

\section{LITERATUR}

Eismann, W. (1988): Zum Problem der Äquivalenz von Phraseologismen. In: G. Greciano (Hg.): Europhras '88. Phraselogie contrastive. Actes du colloque international. Klingenthal, Strasbourg, 83-93.

Fleischer, W. (1982): Phraseologie der deutschen Gegenwartssprache. Leipzig.

Földes, C. (1991): Farbbezeichnungen als phraseologische Strukturkomponente im Deutschen, Russischen und Ungarischen. In: Ch. Palm (Hg.): Europhras '90, 77-88.

Heller, E. (1989): Wie Farben wirken. Farbpsychologie, Farbsymbolik, kreative Farbgestaltung. Hamburg.

Hessky, R. (1980): Zur kontrastiven Untersuchung idiomatischer Wendungen. In: J. Juhasz (Hg.): Konfrontative Studien ungarisch-deutsch. Budapest, 65-75.

Koch, W. (1931): Psychologische Farbenlehre. Die sinnlich-sittliche Wirkung der Farben. Halle.

Majkowska, G. (1996) : Funkcje frazeologizmów w tekstach publicystycznych. In: A.M. Lewicki (Hg.): Problemy frazeologii europejskiej. Warszawa, 39-47.

Michera, W. (1987): Kolory w procesie symbolizacji. In: T. Kostyrko (Hg.): Symbol i poznanie. W poszukiwaniu koncepcji integrujacej. Warszawa. 86-106.

Lipiński, K. (2000): Vademecum thumacza. Kraków.

Labno-Falęcka, E. (1995): Phraseologie und Übersetzen. Frankfurt/M. - Berlin - Bern - New York - Paris - Wien.

Schatte, Cz. (1999): Phraseologismen als sekundäre Nomination in deutschen und polnischen literarischen Texten. In: B. Döring; A. Feine; W. Schellenberg (Hg.): Über Sprachhandeln im Spannungsfeld von Reflektieren und Benennen. Frankfurt/M. - Berlin - Bern - New York - Paris - Wien, 215-226.

Wawrzyniak, Z. (1989): Sinn und Form der Translation. In: A. Kątny (Hg.): Studien zur kontrastiven Linguistik und literarischen Übersetzung. Frankfurt/M. - Bern - New York - Paris, 199-209.

Zybatow, L. (1998): Übersetzen von Phraseologismen oder was bringt die kognitive Linguistik dem Übersetzer? In: J. Wirrer (Hg.): Phraseologismen in Text und Kontext. Bielefeld, 149168. 